\title{
Adaptive Energy Harvesting Relaying Protocol for Two-Way Half-Duplex System Network over Rician Fading Channels
}

\author{
Tan N. Nguyen, ${ }^{1,2}$ Tran Hoang Quang Minh ${ }^{(D)}{ }^{3}$ \\ Phuong T. Tran $\left(\mathbb{B},{ }^{1}\right.$ and Miroslav Voznak $\mathbb{1}^{2}{ }^{2}$ \\ ${ }^{1}$ Wireless Communications Research Group, Faculty of Electrical \& Electronics Engineering, Ton Duc Thang University, \\ 19 Nguyen Huu Tho Street, Ho Chi Minh City, Vietnam \\ ${ }^{2}$ Faculty of Electrical Engineering and Computer Science, Technical University of Ostrava, Ostrava, Czech Republic \\ ${ }^{3}$ Optoelectronics Research Group, Faculty of Electrical \& Electronics Engineering, Ton Duc Thang University, \\ 19 Nguyen Huu Tho Street, Ho Chi Minh City, Vietnam
}

Correspondence should be addressed to Phuong T. Tran; tranthanhphuong@tdt.edu.vn

Received 11 November 2017; Revised 23 January 2018; Accepted 22 March 2018; Published 26 April 2018

Academic Editor: Juan F. Valenzuela-Valdés

Copyright (C) 2018 Tan N. Nguyen et al. This is an open access article distributed under the Creative Commons Attribution License, which permits unrestricted use, distribution, and reproduction in any medium, provided the original work is properly cited.

We investigate the system performance of a two-way amplify-and-forward (AF) energy harvesting relay network over the Rician fading environment. For details, the delay-limited (DL) and delay-tolerant (DT) transmission modes are proposed and investigated when both energy and information are transferred between the source node and the destination node via a relay node. In the first stage, the analytical expressions of the achievable throughput, ergodic capacity, the outage probability, and symbol error ratio (SER) were proposed, analyzed, and demonstrated. After that, the closed-form expressions for the system performance are studied in connection with all system parameters. Moreover, the analytical results are also demonstrated by Monte Carlo simulation in comparison with the closed-form expressions. Finally, the research results show that the analytical and the simulation results agree well with each other in all system parameters.

\section{Introduction}

In the last decades, the Internet of Things (IoT) is a too hot research area over the world. With its potential to significantly influence all aspects of our daily lives, IoT is expected to have a significant impact on businesses by automating some processes and improving the control of many environment variables [1-6]. However, the large-scale realization of IoT is not easy, and numerous challenges need to be overcome before this concept is widely accepted. Energy limitation is the most significant problem in IoT technology revolution for long-term operation of wireless networks. In the beginning, this problem is solved by prolonging the lifetime or by battery recharging/replacement, which may not always be a perspective and practical solution for the wireless networks. In the last few years, energy harvesting has been considered as a prospective solution for prolonging the lifetime of wireless devices and a promising technique for IoT shortly. The sources for energy harvesting are an extensive range such as solar, wind, radio frequency (RF), and vibration. However, RF energy harvesting provides critical advantages over other energy collection methods due to its predictable and stable nature, small form factor, and low cost. The wireless powered communication network (WPCN), where network devices harvest energy from the signals transmitted by RF energy sources in the first step and then utilize this harvested energy for their communication needs, is the primary direction in the RF energy harvesting. In the last decade, many research papers focused on the WPCN and how to improve its efficiency. This concept of a tradeoff between $\mathrm{EH}$ and information transmission in WPCN was proposed and investigated in [7] and extended in [8]. Moreover, the concept of partial network level cooperation for $\mathrm{EH}$ networks was presented in detail in [9], and in [10] wireless EH and information transfer in cognitive relay networks was intensely analyzed. From this point of 
view, a wireless powered communication network (WPCN) with RF energy harvesting is the hot research area in tour decade.

In WPCN, the two traditional time switching (TSP) and power splitting (PSP) protocols have been intensively studied in the literature, and many from these studies have compared the system performance of the two protocols under different scenarios [11-15]. From these studies, the TSP and PSP protocols have some drawbacks; for instance, TSP has to lose some information while it switches to the harvesting mode and PSP has a low coverage area. Furthermore, PSP requires a complicated hardware structure to make sure that a proper portion of energy from source signal is extracted for energy harvesting. In contrast, TSP can simplify the hardware at the expense of the throughput or achievable rate of the system. Based on the fact that both TSP and PSP protocols have their drawbacks, the prospective idea is to combine these two protocols to get the best out of them. This solution can be obtained in this paper by using an adaptive relaying protocol. Also, the adaptive relaying network is investigated for the two-way half-duplex system over the Rician fading channel. This model is more used in practice in comparison with the widespread Raleigh fading channel [16-20]

In this paper, the system performance (in terms of the achievable throughput, ergodic capacity, the outage probability, and symbol errors ratio (SER) of a two-way amplify-andforward (AF) adaptive relaying network over Rician fading channel) is investigated in detail. In this model, the energy and information are transferred between the destination node and source node via relay node by DL and DT transmission modes. In the first step, the analytical mathematical expressions of the achievable throughput, outage probability, ergodic capacity, and SER were proposed and demonstrated for both DL and DT transmission modes. In the first stage, the analytical expressions of the achievable throughput, ergodic capacity, the outage probability, and symbol error ratio (SER) were proposed, analyzed, and demonstrated. After that, the closed-form expressions for the system performance are studied in connection with all system parameters. Moreover, the analytical results are also demonstrated by Monte Carlo simulation in comparison with the closed-form expressions. Finally, the research results show that the analytical and the simulation results agree well with each other in all system parameters.

The research results provided the practical solution for the progress of the WPCN in the near future. The main contributions are summarized as follows:

(1) The system model of the adaptive relaying network for the two-way half-duplex system over the Rician fading channel is proposed in both DT and DL transmission modes.

(2) The closed-form expressions of the outage probability, achievable throughput, ergodic capacity, and especially SER of the system model for the DL and DT transmission modes are proposed and derived.

(3) Use Monte Carlo simulation to clear the analysis results and compare the system performance of all TS, PS, and adaptive protocols.

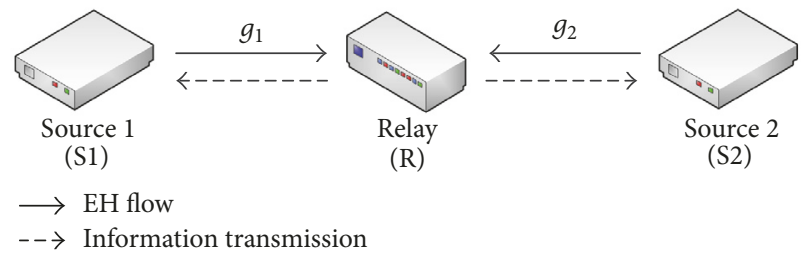

Figure 1: System model.

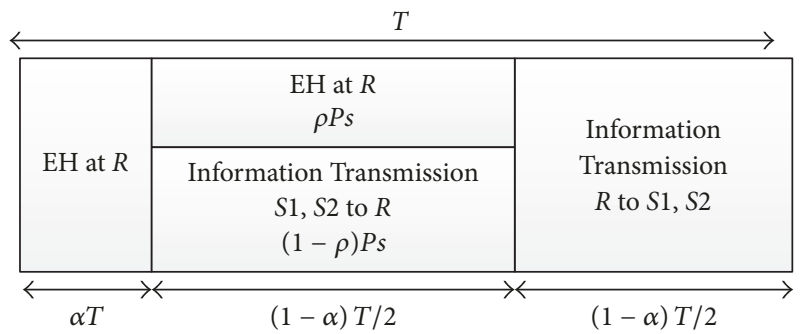

FIGURE 2: The energy harvesting and information processing by the adaptive relaying protocol.

The structure of the remainder of this paper is proposed as follows. Section 2 shows the system model of two-way AR adaptive relaying network over Rician fading channel in both DT and DL transmission modes. Section 3 proposed the system performance of the model system in detail. Section 4 presents the numerical results and some discussions. Finally, conclusions of the paper are proposed in Section 5.

\section{System Model}

In this section, amplify-and-forward (AF) adaptive relaying protocol for two-way half-duplex system network over Rician fading channel is presented in Figure 1. In Figure 1, the information and energy are transferred between the sources $(S 1, S 2)$ and the relay $(R)$ in two-way with the half-duplex system. The energy harvesting and information processing of the adaptive relaying network are proposed in Figure 2. The all-time interval for the energy harvesting and information processing is denoted by $T$, which is divided into three intervals. The first portion of time $\alpha T$ is used for energy harvesting at $R$ from the sources $S 1$ and $S 2$. In the second interval, whose length is $(1-\alpha) T / 2$, the source signal is divided into two streams. During this interval, a fraction of the power $\rho P_{S}$ is used for energy harvesting from the sources $S 1$ and $S 2$ signal by the relay node, and a fraction $(1-\rho) P_{S}$ is used for decoding the information signal sent from the source nodes. The remaining interval of the length $(1-\alpha) T / 2$ is used for information forwarding from the relay to the destination nodes $S 1$ and $S 2$. Obviously, $0 \leq \alpha \leq 1$ and $0 \leq \rho \leq 1$. If $\alpha=0$, this scheme becomes PSP. If $\rho=0$, then it becomes the TSP protocol.

2.1. Energy Harvesting. In the energy harvesting phase at the relay node, the received signal at the relay node can be calculated as 


$$
y_{r}=g_{1} x_{s_{1}}+g_{2} x_{s_{2}}+n_{r} .
$$

In this equation, $x_{s_{1,2}}$ is the energy-transmitted signal from sources $S 1$ and $S 2$ with $E\left\{\left|x_{s_{1}}\right|^{2}\right\}=E\left\{\left|x_{s_{2}}\right|^{2}\right\}=P_{s}$, and $n_{r}$ is the zero-mean additive white Gaussian noise (AWGN) with variance $N_{0}$. Here $E\{\cdot\}$ denotes the expectation operation, and $g_{1} g_{2}$ are the Rician distribution factors.

The energy harvested at the relay node is the combination of two components: the first one is the received energy during the first interval $\alpha T$ (from TSR protocol), while the second one comes from the PSR interval $(1-\alpha) T / 2$. The amount of harvested energy can be calculated as

$$
\begin{aligned}
E_{r}= & \left(\eta_{\alpha} P_{s}\left|g_{1}\right|^{2}+\eta_{\alpha} P_{s}\left|g_{2}\right|^{2}\right) \alpha T \\
& +\left(\eta_{\rho} \rho P_{s}\left|g_{1}\right|^{2}+\eta_{\rho} \rho P_{s}\left|g_{2}\right|^{2}\right) \frac{(1-\alpha) T}{2} \\
= & {\left[\eta_{\alpha} \alpha T+\frac{\eta_{\rho} \rho(1-\alpha) T}{2}\right]\left(P_{s}\left|g_{1}\right|^{2}+P_{s}\left|g_{2}\right|^{2}\right), }
\end{aligned}
$$

where $\eta_{\alpha}, \eta_{\rho}$ represent the EH efficiency of the two stages, TS and PS, respectively, $0<\eta_{\alpha}<1$, and $0<\eta_{\rho}<1$.

Finally, the received power at the relay node can be computed as

$$
\begin{aligned}
P_{r} & =\frac{E_{r}}{(1-\alpha) T / 2} \\
& =\frac{\left[\eta_{\alpha} \alpha T+\eta_{\rho} \rho(1-\alpha) T / 2\right]\left(P_{s}\left|g_{1}\right|^{2}+P_{s}\left|g_{2}\right|^{2}\right)}{(1-\alpha) T / 2} \\
& =\left[\frac{2 \eta_{\alpha} \alpha}{1-\alpha}+\eta_{\rho} \rho\right]\left(P_{s}\left|g_{1}\right|^{2}+P_{s}\left|g_{2}\right|^{2}\right), \\
P_{r} & =\kappa\left(P_{s}\left|g_{1}\right|^{2}+P_{s}\left|g_{2}\right|^{2}\right),
\end{aligned}
$$

where we denote $\kappa=\left[2 \eta_{\alpha} \alpha /(1-\alpha)+\eta_{\rho} \rho\right]$.

2.2. Information Transmission. In this subsection, the information transmission phase is considered. This interval time is divided into two subintervals. In the first interval, the received signal at the relay can be expressed as

$$
y_{r}=\sqrt{1-\rho} g_{1} x_{s_{1}}+\sqrt{1-\rho} g_{2} x_{s_{2}}+n_{r},
$$

where $n_{r}$ denotes the AWGN noise at the relay node.

In research model, the amplify-and-forward protocol is used. Moreover, the received signal at relay node (which is amplified by a factor $\beta$ ) is proposed by

$$
\beta=\frac{x_{r}}{y_{r}}=\sqrt{\frac{P_{r}}{(1-\rho) P_{s}\left(\left|g_{1}\right|^{2}+\left|g_{2}\right|^{2}\right)+N_{0}}} .
$$

During the second interval, the sources $S 1$ and $S 2$ are receiving the forwarded signal from the relay. Now, because of the symmetry of $S 1$ and $S 2$, we first provide the expression of $y_{1}$ for the source $S 1$. The received signal at the source $S 1$ is given by

$$
y_{1}=g_{1} x_{r}+n_{1}
$$

Here $n_{1}$ is the additive white Gaussian noise (AWGN) at $S_{1}$ with zero mean and variance $N_{0}$. Substituting (4) in (6), (6) could be changed to

$$
\begin{aligned}
y_{1} & =g_{1} x_{r}+n_{1}=g_{1} \beta y_{r}+n_{1} \\
& =g_{1} \beta\left[\sqrt{1-\rho} g_{1} x_{s_{1}}+\sqrt{1-\rho} g_{2} x_{s_{2}}+n_{r}\right]+n_{1} .
\end{aligned}
$$

In (7) $S 1$ wants to extract $S 2$ from $y_{1}$. Since it knows its own transmitted symbol $S 1$, it can perfectly eliminate the corresponding self-interference term $\beta \sqrt{1-\rho}\left|g_{1}\right|^{2} x_{s_{1}}$. After that, we have

$$
y_{1}=\underbrace{g_{1} \beta \sqrt{1-\rho} g_{2} x_{s_{2}}}_{\text {signal }}+\underbrace{g_{1} \beta n_{r}+n_{1}}_{\text {noise }} .
$$

Now, the end-to-end signal-to-noise ratio (SNR) at the source node $S 1$ can be calculated by

$$
\begin{aligned}
\gamma_{1} & =\frac{E\left\{\mid \text { signal }\left.\right|^{2}\right\}}{E\left\{\mid \text { noise }\left.\right|^{2}\right\}}=\frac{\beta^{2}(1-\rho)\left|g_{1}\right|^{2}\left|g_{2}\right|^{2} P_{s}}{\beta^{2}\left|g_{1}\right|^{2} N_{0}+N_{0}} \\
& =\frac{(1-\rho)\left|g_{1}\right|^{2}\left|g_{2}\right|^{2} P_{s}}{\left|g_{1}\right|^{2} N_{0}+N_{0} / \beta^{2}} .
\end{aligned}
$$

Replacing $\beta$ and $P_{r}$ in (9) we have

$\gamma_{1}$

$$
\begin{aligned}
& =\frac{(1-\rho)\left|g_{1}\right|^{2}\left|g_{2}\right|^{2} P_{s}}{\left|g_{1}\right|^{2} N_{0}+N_{0}\left[(1-\rho) P_{s}\left(\left|g_{1}\right|^{2}+\left|g_{2}\right|^{2}\right)+N_{0}\right] / P_{r}} \\
& =\frac{(1-\rho)\left|g_{1}\right|^{2}\left|g_{2}\right|^{2} P_{s}}{\left|g_{1}\right|^{2} N_{0}+N_{0}(1-\rho) / \kappa+N_{0}^{2} / P_{r}} .
\end{aligned}
$$

Because $N_{0} \ll P_{r}$ and we denote $\gamma_{0}=P_{s} / N_{0}$, (10) can be reduced to

$$
\begin{aligned}
\gamma_{1} & \approx \frac{(1-\rho)\left|g_{1}\right|^{2}\left|g_{2}\right|^{2} P_{s}}{\left|g_{1}\right|^{2} N_{0}+N_{0}(1-\rho) / \kappa} \\
& =\frac{(1-\rho)\left|g_{1}\right|^{2}\left|g_{2}\right|^{2} \gamma_{0}}{\left|g_{1}\right|^{2}+(1-\rho) / \kappa}, \\
\gamma_{1} & =\frac{(1-\rho) \varphi_{1} \varphi_{2} \gamma_{0}}{\varphi_{1}+(1-\rho) / \kappa},
\end{aligned}
$$

where $\varphi_{1}=\left|g_{1}\right|^{2}$ and $\varphi_{2}=\left|g_{2}\right|^{2}$.

By the same way, the end-to-end signal-to-noise ratio of $\gamma_{2}$ at the source node $S 2$ is also given by

$$
\gamma_{2}=\frac{(1-\rho) \varphi_{1} \varphi_{2} \gamma_{0}}{\varphi_{2}+(1-\rho) / \kappa}
$$

More details of the analytical mathematical model for delay-limited (DL) transmission and delay-tolerant (DT) transmission modes of the two-way AF relaying system are presented in the following sections [15-22]. 


\section{The System Performance}

Based on the system model on above section, the system performance of the relay network is presented, analyzed, and demonstrated for delay-limited (DL) transmission and delaytolerant (DT) transmission in detail in this section [7-12]. In addition, the outage probability and throughput performance of the proposed system for delay-limited transmission mode and the ergodic capacity of the system for delay-tolerant mode are proposed and derived.

\subsection{Delay-Limited (DL) Transmission}

3.1.1. Outage Probability. The probability density function (PDF) of random variable (RV) $\varphi_{i}$, where $i=1,2$, can be calculated by [21]

$$
\begin{aligned}
& f_{\varphi_{i}}(x) \\
& \quad=\frac{(K+1) e^{-K}}{\lambda_{i}} e^{-(K+1) x / \lambda_{i}} I_{0}\left(2 \sqrt{\frac{K(K+1) x}{\lambda_{i}}}\right),
\end{aligned}
$$

where $\lambda_{i}$ is the mean value of $\operatorname{RV} \varphi_{i}$, where $i=1,2$, respectively. $K$ is the Rician $K$-factor defined as the ratio of the power of the line-of-sight (LOS) component to the scattered components, and $I_{0}(\cdot)$ is the zeroth order modified Bessel function of the first kind.

Using $a=(K+1) e^{-K} / \lambda_{i}, b=(K+1) / \lambda_{i}$, and $I_{0}(x)=$ $\sum_{l=0}^{\infty} x^{2 l} / 2^{2 l}(l !)^{2}[23],(14)$ can be rewritten as follows:

$$
f_{\varphi_{i}}(x)=a \sum_{l=0}^{\infty} \frac{(b K)^{l}}{(l !)^{2}} x^{l} e^{-b x} .
$$

The cumulative density function (CDF) of $\operatorname{RV} \varphi_{i}$, where $i=1,2$, can be computed as in [22]. Here, we assume that $\lambda_{1}=\lambda_{2}$ :

$$
F_{\varphi_{i}}(\varsigma)=\int_{0}^{\varsigma} f_{\varphi_{i}}(x) d x=1-\frac{a}{b} \sum_{l=0}^{\infty} \sum_{m=0}^{l} \frac{K^{l} b^{m}}{l ! m !} \varsigma^{m} e^{-b \varsigma} .
$$

Hence, the outage probability is determined by

$$
\begin{aligned}
P_{\text {out } \_1} & =F_{\gamma_{1}}\left(\gamma_{\text {th }}\right)=\operatorname{Pr}\left(\gamma_{1}<\gamma_{\text {th }}\right) \\
& =\operatorname{Pr}\left(\frac{(1-\rho) \varphi_{1} \varphi_{2} \gamma_{0}}{\varphi_{1}+(1-\rho) / \kappa}<\gamma_{\text {th }}\right) .
\end{aligned}
$$

Assume that the source transmits at a constant rate $R$. Let $\gamma_{\text {th }}=2^{R}-1$ be the lower threshold for SNR at both relay and source nodes. Then the outage probability can reformulate as

$$
\begin{aligned}
& P_{\text {out } \_1}=\int_{0}^{\infty} \operatorname{Pr}\left(\varphi_{2}<\frac{\varphi_{1} \gamma_{\mathrm{th}}+\gamma_{\mathrm{th}}((1-\rho) / \kappa)}{\varphi_{1} \gamma_{0}(1-\rho)} \mid \varphi_{1}\right) \\
& \cdot f_{\varphi_{1}}\left(\varphi_{1}\right) d \varphi_{1} \\
& =\int_{0}^{\infty} F_{\varphi_{2}}\left(\frac{\varphi_{1} \gamma_{\mathrm{th}}+\gamma_{\mathrm{th}}((1-\rho) / \kappa)}{\varphi_{1} \gamma_{0}(1-\rho)}\right) \\
& \cdot f_{\varphi_{1}}\left(\varphi_{1}\right) d \varphi_{1} .
\end{aligned}
$$

Combining (18) with (15) and (16) we have

$$
\begin{aligned}
& P_{\text {out_1 }}=1 \\
& -\int_{0}^{\infty} \frac{a}{b} \sum_{l=0}^{\infty} \sum_{m=0}^{l} \frac{K^{l} b^{m}}{l ! m !}\left[\frac{\varphi_{1} \gamma_{\text {th }}+\gamma_{\text {th }}((1-\rho) / \kappa)}{\varphi_{1} \gamma_{0}(1-\rho)}\right]^{m} \\
& \quad \times e^{-b\left[\varphi_{1} \gamma_{\text {th }}+\gamma_{\text {th }}((1-\rho) / \kappa) / \varphi_{1} \gamma_{0}(1-\rho)\right]} \\
& \quad \times a \sum_{l=0}^{\infty} \frac{(b K)^{l}}{(l !)^{2}} \varphi_{1}^{l} e^{-b \varphi_{1}} d \varphi_{1}, \\
& P_{\text {out_ } 1}=1 \\
& \quad-\int_{0}^{\infty} \frac{a^{2}}{b} \sum_{l=0}^{\infty} \sum_{k=0}^{\infty} \sum_{m=0}^{l} \frac{K^{l+k} b^{m+k}}{l ! m !(k !)^{2}}\left[\frac{\gamma_{\text {th }}}{\gamma_{0}(1-\rho)}+\frac{\gamma_{\text {th }}}{\kappa \varphi_{1} \gamma_{0}}\right]^{m} \\
& \quad \times e^{-b \gamma_{\text {th }} / \gamma_{0}(1-\rho)} \times e^{-b \gamma_{\text {th }} / \kappa \varphi_{1} \gamma_{0}} \times \varphi_{1}^{k} e^{-b \varphi_{1}} d \varphi_{1} .
\end{aligned}
$$

Now by applying the equation $(x+y)^{m}=$ $\sum_{n=0}^{m}\left(\begin{array}{c}m \\ n\end{array}\right) x^{m-n} y^{n}$ to (20), the outage probability can be demonstrated as follows:

$$
\begin{aligned}
& P_{\text {out_1 }}=1-\int_{0}^{\infty} \frac{a^{2}}{b} \sum_{l=0}^{\infty} \sum_{k=0}^{\infty} \sum_{m=0}^{l} \frac{K^{l+k} b^{m+k} \gamma_{\mathrm{th}}^{m}}{l ! m !(k !)^{2} \gamma_{0}^{m}} \sum_{n=0}^{m}\left(\begin{array}{c}
m \\
n
\end{array}\right)\left(\frac{1}{1-\rho}\right)^{m-n} \\
& \cdot\left(\frac{1}{\kappa \varphi_{1}}\right)^{n} \times e^{-b \gamma_{\mathrm{th}} / \gamma_{0}(1-\rho)} \times e^{-b \gamma_{\mathrm{th}} / \kappa \varphi_{1} \gamma_{0}} \times \varphi_{1}^{k} e^{-b \varphi_{1}} d \varphi_{1}, \\
& P_{\text {out_1 }}=1-a^{2} \\
& \quad \times e^{-b \gamma_{\mathrm{th}} / \gamma_{0}(1-\rho)} \sum_{l=0}^{\infty} \sum_{k=0}^{\infty} \sum_{m=0}^{l} \sum_{n=0}^{m} \frac{K^{l+k} b^{m+k-1} \gamma_{\mathrm{th}}^{m}}{l ! n !(m-n) !(k !)^{2} \gamma_{0}^{m}(1-\rho)^{m-n} \kappa^{n}} \\
& \quad \times \int_{0}^{\infty} \varphi_{1}^{k-n} \times e^{-b \gamma_{\mathrm{th}} / \kappa \varphi_{1} \gamma_{0}} \times e^{-b \varphi_{1}} d \varphi_{1} .
\end{aligned}
$$

Using table of integrals equation [3.471,9] in [23], (22) can reformulated as

$$
\begin{aligned}
P_{\text {out_1 }}= & -2 a^{2} \times e^{-b \gamma_{\mathrm{th}} / \gamma_{0}(1-\rho)} \sum_{l=0}^{\infty} \sum_{k=0}^{\infty} \sum_{m=0}^{l} \sum_{n=0}^{m} \frac{K^{l+k} b^{m+k-1}}{l ! n !(m-n) !(k !)^{2}(1-\rho)^{m-n} \kappa^{(k+n+1) / 2}} \times\left(\frac{\gamma_{\text {th }}}{\gamma_{0}}\right)^{m+(k-n+1) / 2} \\
& \times K_{k-n+1}\left(2 b \sqrt{\frac{\gamma_{\text {th }}}{\kappa \gamma_{0}}}\right)
\end{aligned}
$$


where $K_{v}(\cdot)$ is the modified Bessel function of the second kind and $v$ th order.
In the same way, the outage probability at the source node $S 2$ is also given by

$$
\begin{aligned}
P_{\text {out } \_2}= & -2 a^{2} \times e^{-b \gamma_{\text {th }} / \gamma_{0}(1-\rho)} \sum_{l=0}^{\infty} \sum_{k=0}^{\infty} \sum_{m=0}^{l} \sum_{n=0}^{m} \frac{K^{l+k} b^{m+k-1}}{l ! n !(m-n) !(k !)^{2}(1-\rho)^{m-n} \kappa^{(k+n+1) / 2}} \times\left(\frac{\gamma_{\text {th }}}{\gamma_{0}}\right)^{m+(k-n+1) / 2} \\
& \times K_{k-n+1}\left(2 b \sqrt{\frac{\gamma_{\text {th }}}{\kappa \gamma_{0}}}\right) .
\end{aligned}
$$

3.1.2. Achievable Throughput. The achievable throughput at the source nodes $S 1$ and $S 2$ can be computed by

$$
\tau_{i}^{\mathrm{DL}}=\left(1-P_{\mathrm{out}_{i}}\right) \times \frac{R}{2} \times(1-\alpha)
$$

where $i \in(1,2)$.

\subsection{Delay-Tolerant (DT) Transmission}

3.2.1. Ergodic Capacity. In this model, the source transfers at any target rate upper bounded by the ergodic capacity.
Hence, the ergodic capacity is given by the following formula:

$$
\begin{aligned}
C_{1} & =E_{g_{1}, g_{2}}\left\{\log _{2}\left(1+\gamma_{1}\right)\right\} \\
C_{1} & =\int_{0}^{\infty} f_{\gamma_{1}}\left(\gamma_{\mathrm{th}}\right) \log _{2}\left(1+\gamma_{\mathrm{th}}\right) d \gamma_{\mathrm{th}} \\
& =\frac{1}{\ln 2} \int_{0}^{\infty} \frac{1-F_{\gamma_{1}}\left(\gamma_{\mathrm{th}}\right)}{1+\gamma_{\mathrm{th}}} d \gamma_{\mathrm{th}} .
\end{aligned}
$$

Using (22) we have

$$
\begin{aligned}
C_{1} & =\frac{1}{\ln 2} \int_{0}^{\infty}\left\{2 a^{2} \sum_{l=0}^{\infty} \sum_{k=0}^{\infty} \sum_{m=0}^{l} \sum_{n=0}^{m} \frac{K^{l+k} b^{m+k-1}}{l ! n !(m-n) !(k !)^{2}(1-\rho)^{m-n} \kappa^{(k+n+1) / 2}\left(1+\gamma_{\mathrm{th}}\right)} \times\left(\frac{\gamma_{\mathrm{th}}}{\gamma_{0}}\right)^{m+(k-n+1) / 2} \times e^{-b \gamma_{\mathrm{th}} / \gamma_{0}(1-\rho)}\right. \\
& \left.\times K_{k-n+1}\left(2 b \sqrt{\frac{\gamma_{\mathrm{th}}}{\kappa \gamma_{0}}}\right)\right\} d \gamma_{\mathrm{th}} .
\end{aligned}
$$

By the same way, the ergodic capacity of the source node $S 2$ is also given by

$$
\begin{aligned}
C_{2} & =\frac{1}{\ln 2} \int_{0}^{\infty}\left\{2 a^{2} \sum_{l=0}^{\infty} \sum_{k=0}^{\infty} \sum_{m=0}^{l} \sum_{n=0}^{m} \frac{K^{l+k} b^{m+k-1}}{l ! n !(m-n) !(k !)^{2}(1-\rho)^{m-n} \kappa^{(k+n+1) / 2}\left(1+\gamma_{\mathrm{th}}\right)} \times\left(\frac{\gamma_{\mathrm{th}}}{\gamma_{0}}\right)^{m+(k-n+1) / 2} \times e^{-b \gamma_{\mathrm{th}} / \gamma_{0}(1-\rho)}\right. \\
& \left.\times K_{k-n+1}\left(2 b \sqrt{\frac{\gamma_{t h}}{\kappa \gamma_{0}}}\right)\right\} d \gamma_{\mathrm{th}} .
\end{aligned}
$$

3.2.2. Achievable Throughput. The achievable throughput at the source nodes $S 1$ and $S 2$ can be computed by

$$
\tau_{i}^{\mathrm{DT}}=C_{i} \times \frac{(1-\alpha)}{2},
$$

where $i \in(1,2)$

3.3. Symbol Error Ratio (SER) Analysis. In this section, the expressions for the symbol error ratio (SER) at the sources $S 1$ and $S 2$ of the two-way AF relaying system are obtained. Thus, we have SER equations as below [24]:

$$
\operatorname{SER}_{i}=E\left[\omega Q\left(\sqrt{2 \theta \gamma_{i}}\right)\right]
$$

where $i \in(1,2)$ and $Q(t)=(1 / \sqrt{2 \pi}) \int_{t}^{\infty} e^{-x^{2} / 2} d x$ is the Gaussian Q-function; $\omega$ and $\theta$ are constants, which is specific for modulation type. Here $(\omega, \theta)=(1,1)$ for BPSK and $(\omega, \theta)=(1,2)$ for QPSK. Before obtaining the SER performance, the distribution function of $\gamma_{i}$ is expected. Then, the SER expression is given in (29) directly regarding outage probability at the source by using integration, as follows:

$$
\begin{aligned}
& \operatorname{SER}_{i}=\frac{\omega \sqrt{\theta}}{2 \sqrt{\pi}} \int_{0}^{\infty} \frac{e^{-\theta x}}{\sqrt{x}} F_{\gamma_{i}}(x) d x, \\
& \operatorname{SER}_{1}=\frac{\omega \sqrt{\theta}}{2 \sqrt{\pi}} \int_{0}^{\infty} \frac{e^{-\theta x}}{\sqrt{x}} F_{\gamma_{1}}(x) d x .
\end{aligned}
$$

Replacing (29) in (32), we have 


$$
\begin{aligned}
& \operatorname{SER}_{1}=\frac{\omega \sqrt{\theta}}{2 \sqrt{\pi}} \int_{0}^{\infty} \frac{e^{-\theta x}}{\sqrt{x}}\left(1-2 a^{2} \times e^{-b x / \gamma_{0}(1-\rho)} \sum_{l=0}^{\infty} \sum_{k=0}^{\infty} \sum_{m=0}^{l} \sum_{n=0}^{m} \frac{K^{l+k} b^{m+k-1}}{l ! n !(m-n) !(k !)^{2}(1-\rho)^{m-n} \kappa^{(k+n+1) / 2} \times\left(\frac{x}{\gamma_{0}}\right)^{m+(k-n+1) / 2}}\right. \\
& \left.\quad \times K_{k-n+1}\left(2 b \sqrt{\frac{x}{\kappa \gamma_{0}}}\right)\right) d x, \\
& \operatorname{SER}_{1}=\frac{\omega \sqrt{\theta}}{2 \sqrt{\pi}} \int_{0}^{\infty} \frac{e^{-\theta x}}{\sqrt{x}} d x-\frac{a^{2} \omega \sqrt{\theta}}{\sqrt{\pi}} \int_{0}^{\infty}\left\{\sum_{l=0}^{\infty} \sum_{k=0}^{\infty} \sum_{m=0}^{l} \sum_{n=0}^{m} \frac{K^{l+k} b^{m+k-1}}{l ! n !(m-n) !(k !)^{2}(1-\rho)^{m-n} \kappa^{(k+n+1) / 2} \gamma_{0}^{m+(k+n+1) / 2} \times x^{(2 m-n+k) / 2}}\right. \\
& \left.\quad \times e^{-x\left[b /(1-\rho) \gamma_{0}+\theta\right]} K_{k-n+1}\left(2 b \sqrt{\frac{x}{\kappa \gamma_{0}}}\right)\right\} d x .
\end{aligned}
$$

Here

$$
\begin{aligned}
I_{1}= & \frac{\omega \sqrt{\theta}}{2 \sqrt{\pi}} \int_{0}^{\infty} \frac{e^{-\theta x}}{\sqrt{x}} d x \\
I_{2}= & \int_{0}^{\infty} x^{(2 m-n+k) / 2} \\
& \times e^{-x\left[b /(1-\rho) \gamma_{0}+\theta\right]} K_{k-n+1}\left(2 b \sqrt{\frac{x}{\kappa \gamma_{0}}}\right) d x
\end{aligned}
$$

$I_{2}$

$$
\begin{aligned}
& =\left[\frac{b}{(1-\rho) \gamma_{0}}+\theta\right]^{-(2 m-n+k+1) / 2} \times \frac{\sqrt{\kappa \gamma_{0}}}{2 b} \\
& \times \Gamma\left(m+\frac{1}{2}\right) \times \Gamma\left(m-n+k+\frac{3}{2}\right) \\
& \times \exp \left[\frac{b^{2}(1-\rho)}{2 \kappa b+2 \kappa \theta(1-\rho) \gamma_{0}}\right] \\
& \times W_{-(2 m-n+k+1) / 2,(k-n+1) / 2}\left[\frac{b^{2}(1-\rho)}{\kappa\left\{b+\theta(1-\rho) \gamma_{0}\right\}}\right] \text {. }
\end{aligned}
$$

$$
I_{1}=\frac{\omega \sqrt{\theta}}{2 \sqrt{\pi}} \times \frac{\sqrt{\pi}}{\sqrt{\theta}}=\frac{\omega}{2} .
$$

Using equation $(6.643,3)$ in table of integrals [23], have

$$
\begin{gathered}
\int_{0}^{\infty} t^{\mu-(1 / 2)} e^{-\alpha t} K_{2 v}(2 \beta \sqrt{t}) d t=\frac{\alpha^{-\mu}}{2 \beta} \Gamma\left(\mu-v+\frac{1}{2}\right) \\
\cdot \Gamma\left(\mu+v+\frac{1}{2}\right) \exp \left(\frac{\beta^{2}}{2 \alpha}\right) W_{-\mu, v}\left(\frac{\beta^{2}}{\alpha}\right),
\end{gathered}
$$

$$
\begin{aligned}
\operatorname{SER}_{1}= & \frac{\omega}{2}-\frac{a^{2} \omega \sqrt{\theta}}{\sqrt{\pi}} \times \sum_{l=0}^{\infty} \sum_{k=0}^{\infty} \sum_{m=0}^{l} \sum_{n=0}^{m} \frac{K^{l+k} b^{m+k-1}}{l ! n !(m-n) !(k !)^{2}(1-\rho)^{m-n} \kappa^{(k+n+1) / 2} \gamma_{0}^{m+(k-n+1) / 2} \times\left[\frac{b}{(1-\rho) \gamma_{0}}+\theta\right]^{-(2 m-n+k+1) / 2}} \\
& \times \frac{\sqrt{\kappa \gamma_{0}}}{2 b} \times \Gamma\left(m+\frac{1}{2}\right) \times \Gamma\left(m-n+k+\frac{3}{2}\right) \times \exp \left[\frac{b^{2}(1-\rho)}{2 \kappa b+2 \kappa \theta(1-\rho) \gamma_{0}}\right] \\
& \times W_{-(2 m-n+k+1) / 2,(k-n+1) / 2}\left[\frac{b^{2}(1-\rho)}{\kappa\left\{b+\theta(1-\rho) \gamma_{0}\right\}}\right]
\end{aligned}
$$

Finally, SER of the source $S 1$ can be calculated by the following equation:

$$
\begin{aligned}
\mathrm{SER}_{1} & =\frac{\omega}{2}-\frac{a^{2} \omega \sqrt{\theta}}{2 \sqrt{\pi}} \\
\times & \sum_{l=0}^{\infty} \sum_{k=0}^{\infty} \sum_{m=0}^{l} \sum_{n=0}^{m} \frac{K^{l+k} b^{m+k-2}}{l ! n !(m-n) !(k !)^{2}(1-\rho)^{m-n} \kappa^{(k+n) / 2} \gamma_{0}^{m+(k-n) / 2}}
\end{aligned}
$$

$$
\begin{aligned}
& \times\left[\frac{b}{(1-\rho) \gamma_{0}}+\theta\right]^{-(2 m-n+k+1) / 2} \times \Gamma\left(m+\frac{1}{2}\right) \\
& \times \Gamma\left(m-n+k+\frac{3}{2}\right) \times \exp \left[\frac{b^{2}(1-\rho)}{2 \kappa b+2 \kappa \theta(1-\rho) \gamma_{0}}\right] \\
& \times W_{-(2 m-n+k+1) / 2,(k-n+1) / 2}\left[\frac{b^{2}(1-\rho)}{\kappa\left\{b+\theta(1-\rho) \gamma_{0}\right\}}\right] .
\end{aligned}
$$


TABLE 1: Simulation parameters.

\begin{tabular}{lcc}
\hline Symbol & Name & Values \\
\hline$\eta_{\alpha}=\eta_{\rho}$ & Energy harvesting efficiency & 0.7 \\
$\lambda_{1}$ & Mean of $\left|g_{1}\right|^{2}$ & 1 \\
$\lambda_{2}$ & Mean of $\left|g_{2}\right|^{2}$ & 1 \\
$K$ & Rician $K$-factor & 3 \\
$\gamma_{\text {th }}$ & SNR threshold & 7 \\
$P_{s} / N_{0}$ & Source power to noise ratio & $0-30 \mathrm{~dB}$ \\
$R$ & Source rate & $3 \mathrm{bit} / \mathrm{s} / \mathrm{Hz}$ \\
\hline
\end{tabular}

In the same way as source $S 1$, the SER of the source $S 2$ can be demonstrated as

$$
\begin{aligned}
\mathrm{SER}_{2} & =\frac{\omega}{2}-\frac{a^{2} \omega \sqrt{\theta}}{2 \sqrt{\pi}} \\
& \times \sum_{l=0}^{\infty} \sum_{k=0}^{\infty} \sum_{m=0}^{l} \sum_{n=0}^{m} \frac{K^{l+k} b^{m+k-2}}{l ! n !(m-n) !(k !)^{2}(1-\rho)^{m-n} \kappa^{(k+n) / 2} \gamma_{0}^{m+(k-n) / 2}} \\
& \times\left[\frac{b}{(1-\rho) \gamma_{0}}+\theta\right]^{-(2 m-n+k+1) / 2} \times \Gamma\left(m+\frac{1}{2}\right) \\
& \times \Gamma\left(m-n+k+\frac{3}{2}\right) \times \exp \left[\frac{b^{2}(1-\rho)}{2 \kappa b+2 \kappa \theta(1-\rho) \gamma_{0}}\right] \\
& \times W_{-(2 m-n+k+1) / 2,(k-n+1) / 2}\left[\frac{b^{2}(1-\rho)}{\kappa\left\{b+\theta(1-\rho) \gamma_{0}\right\}}\right] .
\end{aligned}
$$

\section{Numerical Results and Discussion}

In this research, we use the Monte Carlo simulation to investigate the developed analysis in the previous section. For simplicity, in our simulation model, we assume that the source-relay and relay-destination distances are both normalized to unit value. For the delay-limited transmission mode, the outage probability, achievable throughput, and SER are analyzed in detail. On the other hand, the outage probability, the ergodic capacity, and SER for the delay-tolerant transmission mode are proposed and demonstrated. The main simulation parameters are listed in Table 1.

In Figures 3 and 4, the effect of the ratio on the achievable throughput and the outage probability of the two-way AF relay network system in adaptive relaying (AR), TS, and PS protocols is presented. In this simulation, the DL transmission mode is considered and the ratio $P_{s} / N_{0}$ varied from 0 to $30 \mathrm{~dB}$. For AR protocol, $\alpha$ and $\rho$ are set to 0.5 . From this setting, the parameters for TS and PS protocols are set accordingly to make sure that the information transmission time is equal between 3 methods. From Figures 3 and 4, the analytical and the simulation results match all possible values of $P_{s} / N_{0}$, which confirms that our analysis is reasonable. To be analyzed, the throughput increases and the outage probability decreases when the value of $P_{s} / N_{0}$ increases from 0 to $30 \mathrm{~dB}$. It is also observed that the AR protocol can give better performance than PS protocol.

On the other hand, Figures 5 and 6 plot the influence of the ratio $P_{s} / N_{0}$ on the achievable throughput and the

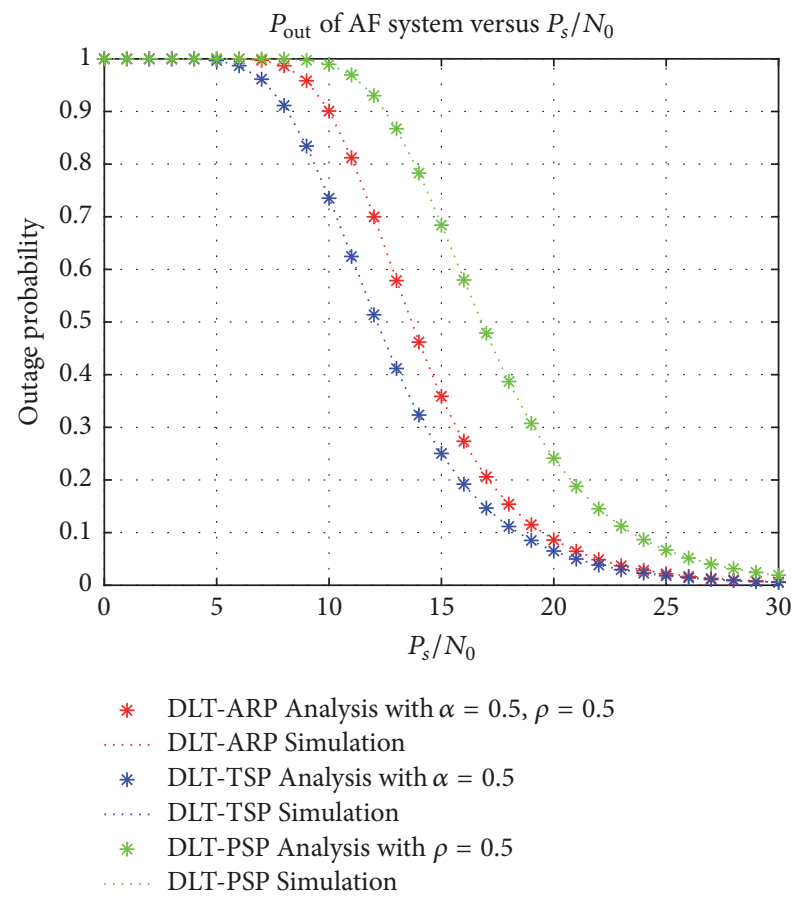

FIgURE 3: The outage probability of the DL transmission mode versus $P_{s} / N_{0}$.

ergodic capacity of the model system in the DT transmission mode. Here, $\alpha$ and $\rho$ are set to 0.5 and the ratio $P_{s} / N_{0}$ increased from 0 to $30 \mathrm{~dB}$. The numerical results show that the throughput and ergodic capacity significantly increased with the increase in the ratio $P_{s} / N_{0}$. Moreover, the simulation results totally agreed with the mathematical analysis from the above section. Furthermore, Figures 7 and 8 illustrate the throughput of the DL and DT transmission modes versus the system factors $\alpha$ and $\rho$. In this cases, $\alpha$ and $\rho$ are set to $0.3,0.5$, and 0.7 in each case. From Figure 7, the throughput increased when $\alpha$ increased to the optimal value at the first step, and after that the throughput crucially decreased. These results are similar to the influence of $\rho$ on the system throughput. Moreover, the simulation results totally agreed with the mathematical analysis from the above section. In addition, the throughput in the DL and DT transmission modes in connection with the ratio $P_{s} / N_{0}$ is presented in Figure 9. After that, SER of the model system is plotted in Figure 10. In all of them, the simulation and analytical mathematical results agreed well with each other. Moreover, the throughput increased, and the SER decreased in AR, TS, and PS protocols, while the ratio $P_{s} / N_{0}$ varied from 0 to $30 \mathrm{~dB}$.

\section{Conclusions}

In this paper, the system performance of a two-way AF relaying network over the Rician fading channel with the delay-limited and delay-tolerant transmission modes has been proposed, analyzed, and demonstrated. In the delaylimited transmission mode, the closed-form expressions of the outage probability, achievable throughput, and SER are 


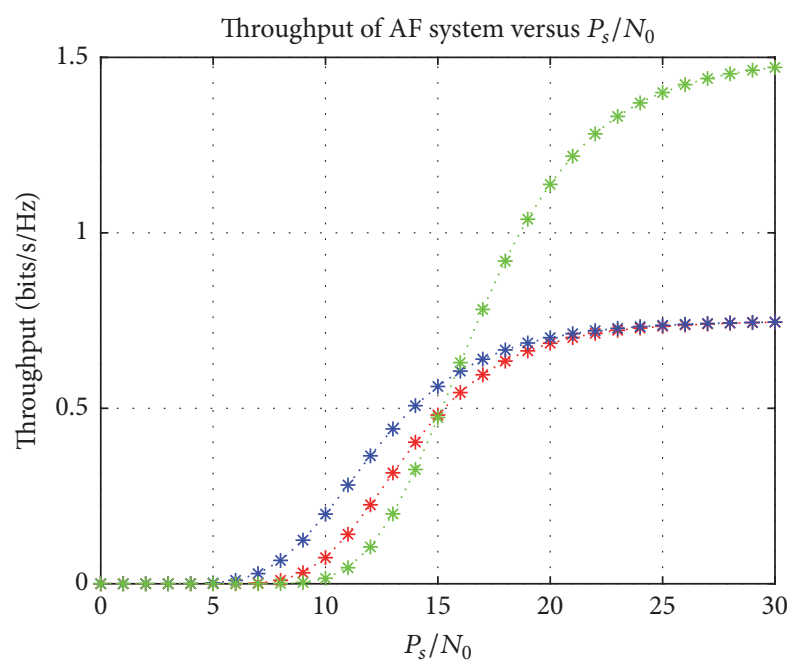

* DLT-ARP Analysis with $\alpha=0.5, \rho=0.5$ DLT-ARP Simulation

* DLT-TSP Analysis with $\alpha=0.5$

.... DLT-TSP Simulation

* DLT-PSP Analysis with $\rho=0.5$ DLT-PSP Simulation

FIGURE 4: The throughput of the DL transmission mode versus $P_{s} / N_{0}$.

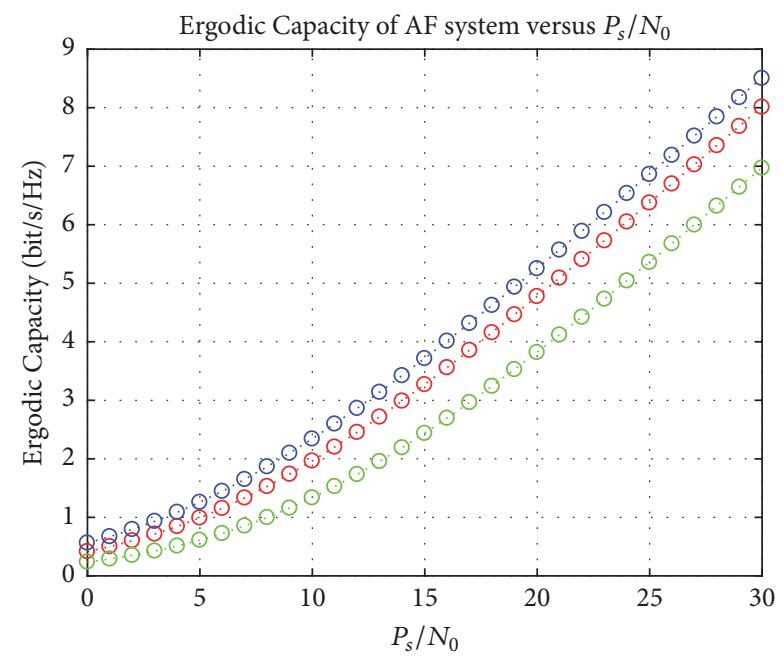

D DTT-ARP Analysis with $\alpha=0.5, \rho=0.5$ DTT-ARP Simulation

○ DTT-TSP Analysis with $\alpha=0.5$

DTT-TSP Simulation

- DTT-PSP Analysis with $\rho=0.5$ DTT-PSP Simulation

FIGURE 5: The ergodic capacity of the DT transmission mode versus $P_{s} / N_{0}$.

proposed and derived. On the other hand, the closed-form expressions of the ergodic capacity, achievable throughput, and SER of the delay-tolerant transmission mode are presented and derived. The numerical results show that the analytical mathematical expression and the simulation results using Monte Carlo method totally match each other.

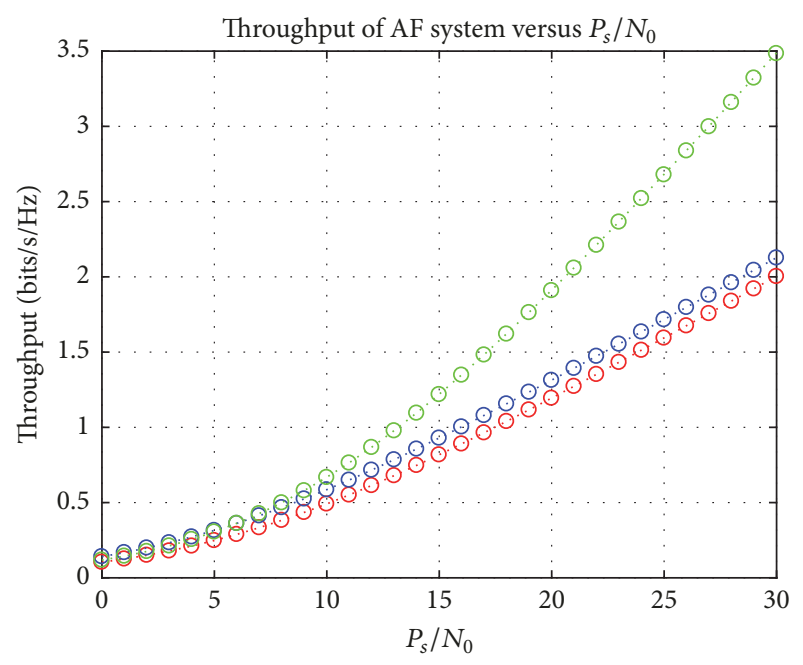
○ DTT-ARP Analysis with $\alpha=0.5, \rho=0.5$ DTT-ARP Simulation
○ DTT-TSP Analysis with $\alpha=0.5$
DTT-TSP Simulation
- DTT-PSP Analysis with $\rho=0.5$
DTT-PSP Simulation

FIGURE 6: The throughput of the DT transmission mode versus $P_{s} / N_{0}$.

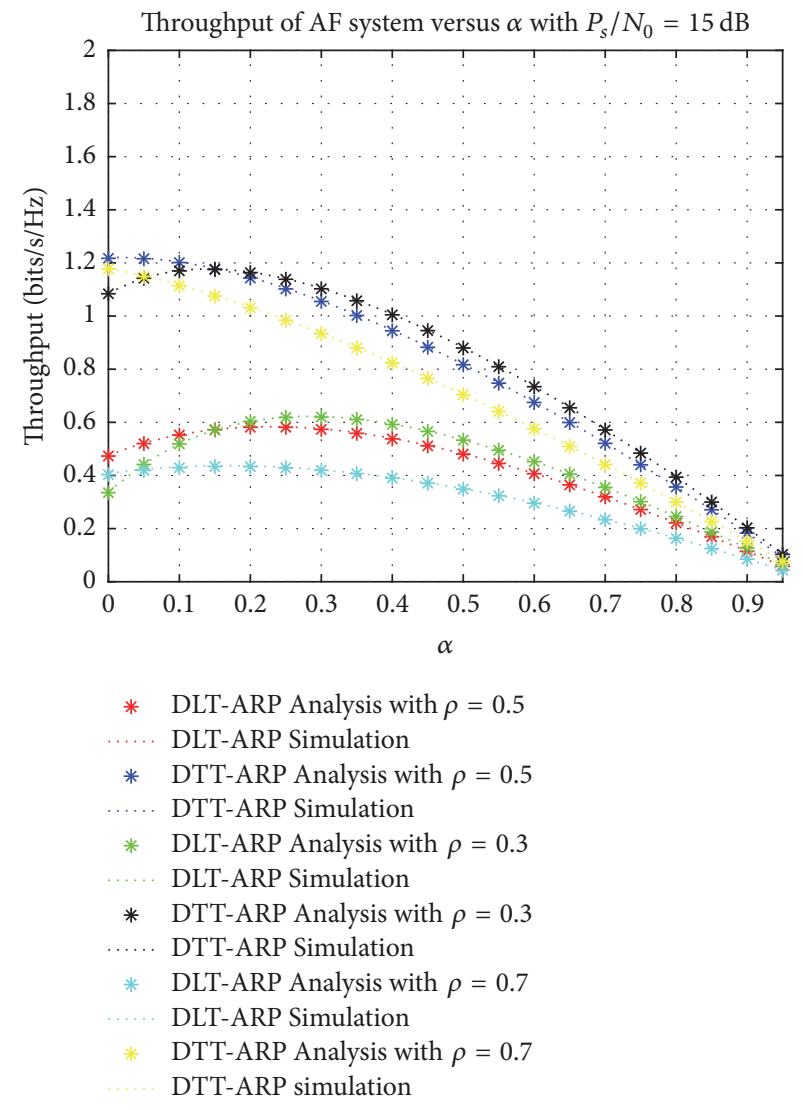

FIgURE 7: The throughput of the DT and DL transmission modes versus $\alpha$. 


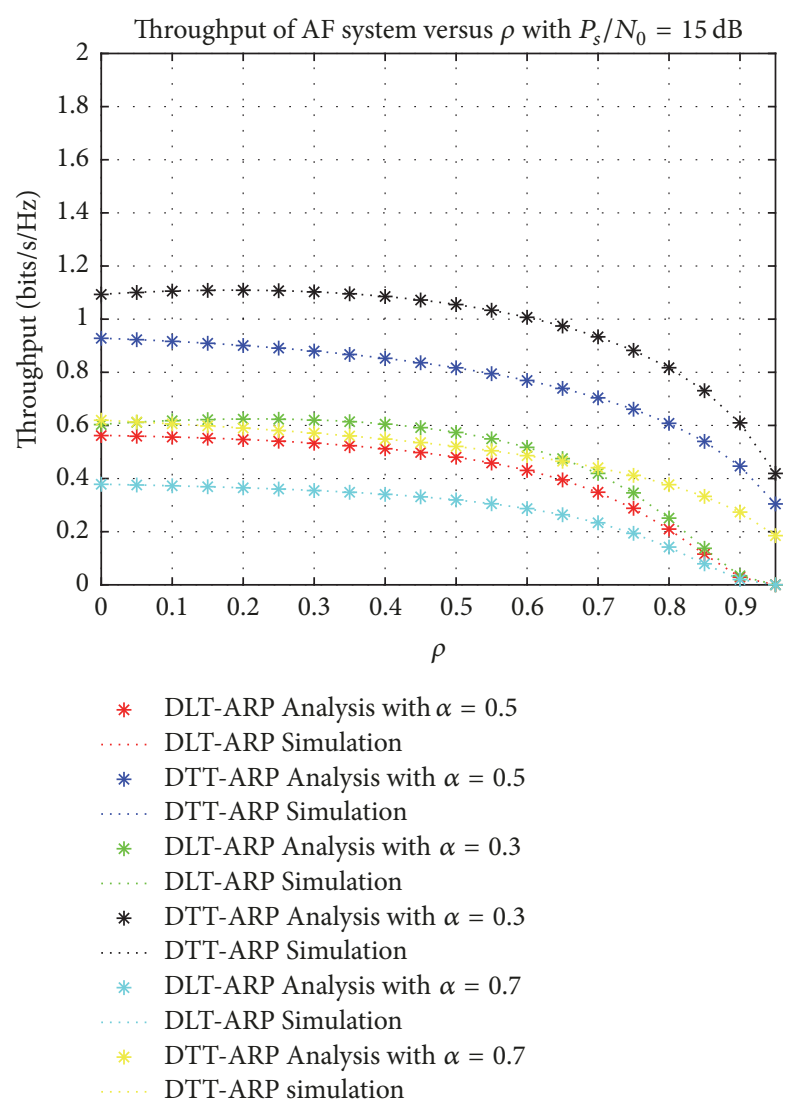

FIgURE 8: The throughput of the DT and DL transmission modes versus $\rho$.

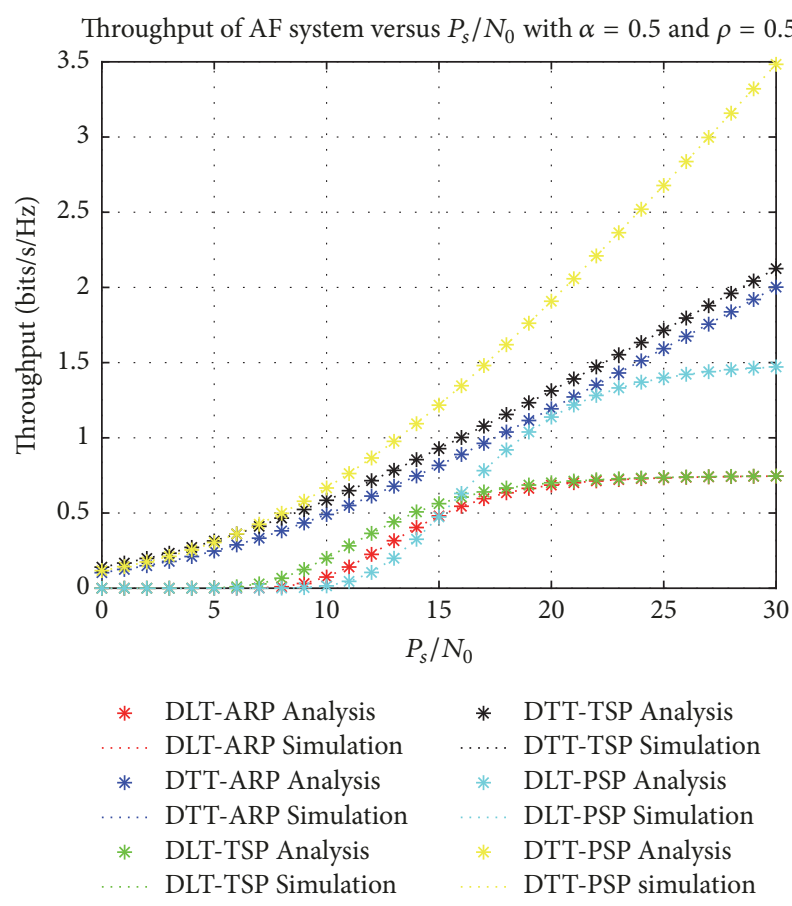

FIGURE 9: The throughput of the DT and DL transmission modes versus $P_{s} / N_{0}$.
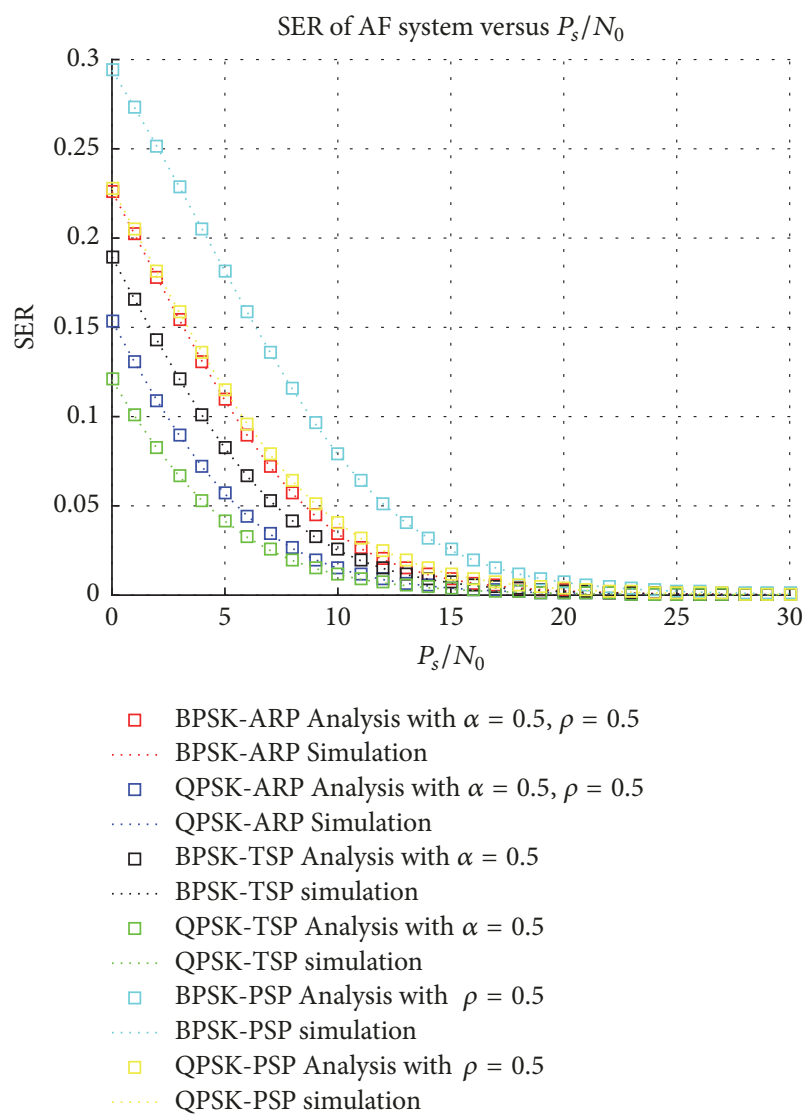

FIgURE 10: SER versus $P_{s} / N_{0}$.

Moreover, this paper has provided practical insights into the effect of various system parameters on the system performance of the two-way AF relaying network over Rician fading channel. The results could provide the prospective solution for the communication network in the near future.

\section{Conflicts of Interest}

The authors declare no conflicts of interest.

\section{Authors' Contributions}

The main contribution of Tan N. Nguyen was to execute performance evaluations by theoretical analysis and simulations, while Phuong T. Tran, Tran Hoang Quang Minh, and Miroslav Voznak worked as the advisors of Tan N. Nguyen in discussing, creating, and advising on performance evaluations together.

\section{Acknowledgments}

The research received financial support from the SGS Grant no. SP2018/59, VSB-Technical University of Ostrava, Czech Republic. 


\section{References}

[1] X. Chen, D. W. K. Ng, and H.-H. Chen, "Secrecy wireless information and power transfer: Challenges and opportunities," IEEE Wireless Communications Magazine, vol. 23, no. 2, pp. 5461, 2016.

[2] S. Bi, C. K. Ho, and R. Zhang, "Wireless powered communication: opportunities and challenges," IEEE Communications Magazine, vol. 53, no. 4, pp. 117-125, 2015.

[3] D. Niyato, D. I. Kim, M. Maso, and Z. Han, "Wireless powered communication networks: research directions and technological approaches," IEEE Wireless Communications, vol. 24, no. 6, pp. 88-97, 2017.

[4] R. Atallah, M. Khabbaz, and C. Assi, "Energy harvesting in vehicular networks: a contemporary survey," IEEE Wireless Communications, vol. 23, no. 2, pp. 70-77, 2016.

[5] K. Tutuncuoglu and A. Yener, "Cooperative energy harvesting communications with relaying and energy sharing," in Proceedings of the IEEE Information Theory Workshop (ITW '13), IEEE, Angeles, Calif, USA, September 2013.

[6] A. A. Nasir, X. Zhou, S. Durrani, and R. A. Kennedy, "Relaying protocols for wireless energy harvesting and information processing," IEEE Transactions on Wireless Communications, vol. 12, no. 7, pp. 3622-3636, 2013.

[7] L. R. Varshney, "Transporting information and energy simultaneously," in Proceedings of the IEEE International Symposium on Information Theory (ISIT '08), pp. 1612-1616, IEEE, Toronto, Canada, July 2008.

[8] X. Zhou, R. Zhang, and C. K. Ho, "Wireless information and power transfer: architecture design and rate-energy tradeoff," in Proceedings of the IEEE Global Communications Conference (GLOBECOM '12), pp. 3982-3987, Anaheim, Calif, USA, December 2012.

[9] M. Kashef and A. Ephremides, "Optimal partial relaying for energy-harvesting wireless networks," IEEE/ACM Transactions on Networking, vol. 24, no. 1, pp. 113-122, 2016.

[10] Z. Wang, Z. Chen, Y. Yao, B. Xia, and H. Liu, "Wireless energy harvesting and information transfer in cognitive two-way relay networks," in Proceedings of the IEEE Global Communications Conference (GLOBECOM '14), pp. 3465-3470, December 2014.

[11] M. Ju, K.-M. Kang, K.-S. Hwang, and C. Jeong, "Maximum transmission rate of PSR/TSR protocols in wireless energy harvesting DF-based relay networks," IEEE Journal on Selected Areas in Communications, vol. 33, no. 12, pp. 2701-2717, 2015.

[12] T. N. Nguyen, D.-T. Do, P. T. Tran, and M. Voznak, "Time switching for wireless communications with full-duplex relaying in imperfect CSI condition," KSII Transactions on Internet and Information Systems, vol. 10, no. 9, pp. 4223-4239, 2016.

[13] Y. Gu and S. Aissa, "RF-based energy harvesting in decodeand-forward relaying systems: ergodic and outage capacities," IEEE Transactions on Wireless Communications, vol. 14, no. 11, pp. 6425-6434, 2015.

[14] Z. Zhou, M. Peng, Z. Zhao, and Y. Li, "Joint power splitting and antenna selection in energy harvesting relay channels," IEEE Signal Processing Letters, vol. 22, no. 7, pp. 823-827, 2015.

[15] L. Liu, R. Zhang, and K. C. Chua, "Wireless information and power transfer: a dynamic power splitting approach," IEEE Transactions on Communications, vol. 61, no. 9, pp. 3990-4001, 2013.

[16] E. Biglieri, J. Proakis, and S. Shamai, "Fading channels: information-theoretic and communications aspects," IEEE Transactions on Information Theory, vol. 44, no. 6, pp. 2619-2692, 1998.
[17] A. H. Abd El-Malek, F. S. Al-Qahtani, T. Q. Duong, S. A. Zummo, and $\mathrm{H}$. Alnuweiri, "MIMO cognitive relay networks with correlated antennas over Rayleigh fading channels," IEEE Transactions on Vehicular Technology, vol. 65, no. 7, pp. 53495363, 2016.

[18] T. T. Duy, G. C. Alexandropoulos, V. T. Tung, V. N. Son, and T. Q. Duong, "Outage performance of cognitive cooperative networks with relay selection over double-Rayleigh fading channels," IET Communications, vol. 10, no. 1, pp. 57-64, 2016.

[19] R. Tao, A. Salem, and K. A. Hamdi, "Adaptive relaying protocol for wireless power transfer and information processing," IEEE Communications Letters, vol. 20, no. 10, pp. 2027-2030, 2016.

[20] T. Li, P. Fan, and K. B. Letaief, "Outage probability of energy harvesting relay-aided cooperative networks over rayleigh fading channel," IEEE Transactions on Vehicular Technology, vol. 65, no. 2, pp. 972-978, 2016.

[21] H. Suraweera, G. K. Karagiannidis, and P. J. Smith, "Performance analysis of the dual-hop asymmetric fading channel," IEEE Transactions on Wireless Communications, vol. 8, no. 6, pp. 2783-2788, 2009.

[22] M. R. Bhatnagar, "On the capacity of decode-and-forward relaying over Rician fading channels," IEEE Communications Letters, vol. 17, no. 6, pp. 1100-1103, 2013.

[23] D. Zwillinger, Ed., Table of Integrals, Series, and Products, Elsevier, 2015.

[24] M. R. McKay, A. J. Grant, and I. B. Collings, "Performance analysis of MIMO-MRC in double-correlated Rayleigh environments," IEEE Transactions on Communications, vol. 55, no. 3, pp. 497-507, 2007. 


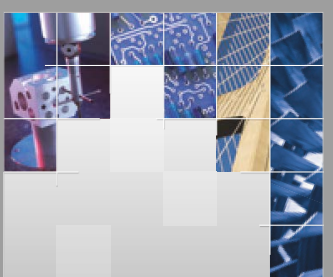

\section{Enfincering}
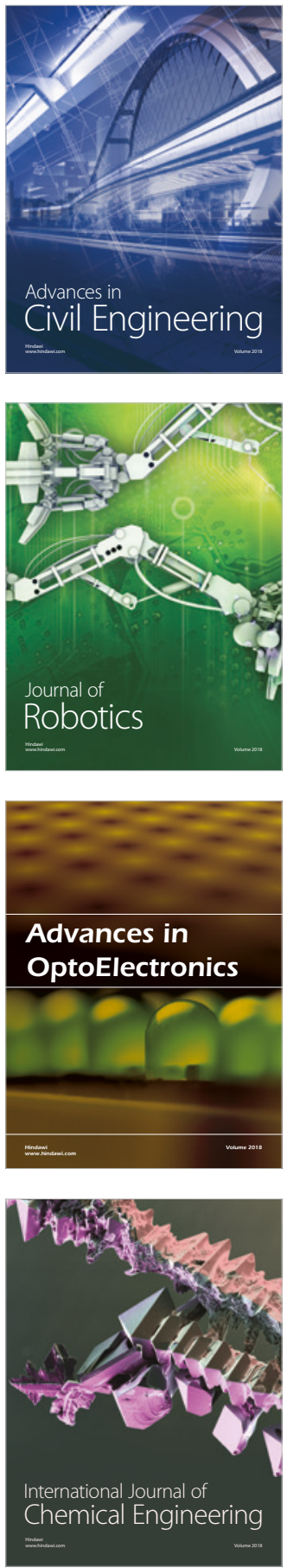

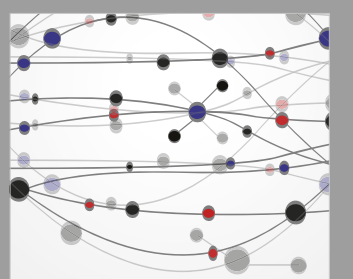

\section{Rotating \\ Machinery}

The Scientific World Journal

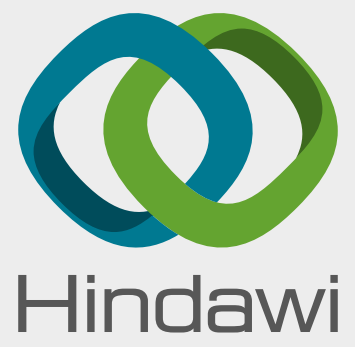

Submit your manuscripts at

www.hindawi.com
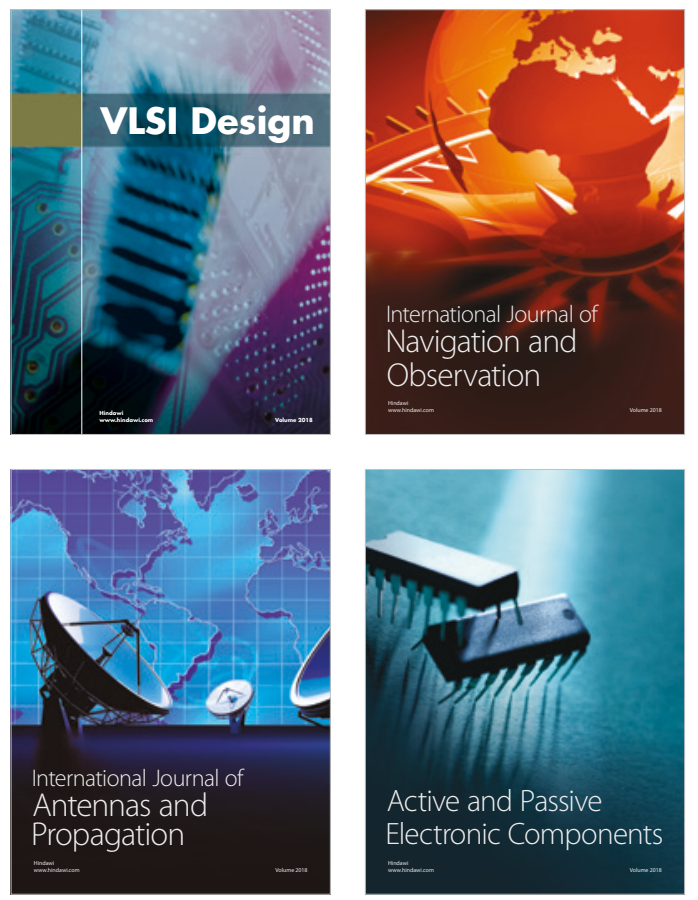
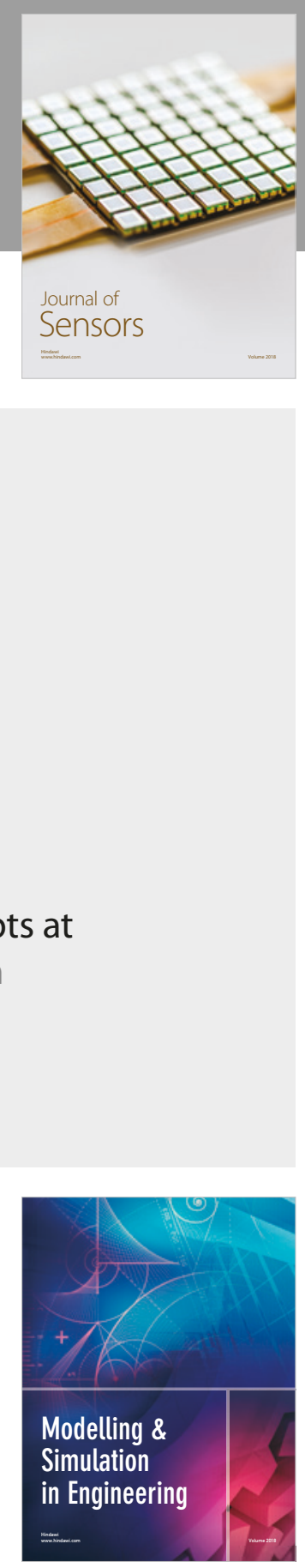

\section{Advances \\ Multimedia}
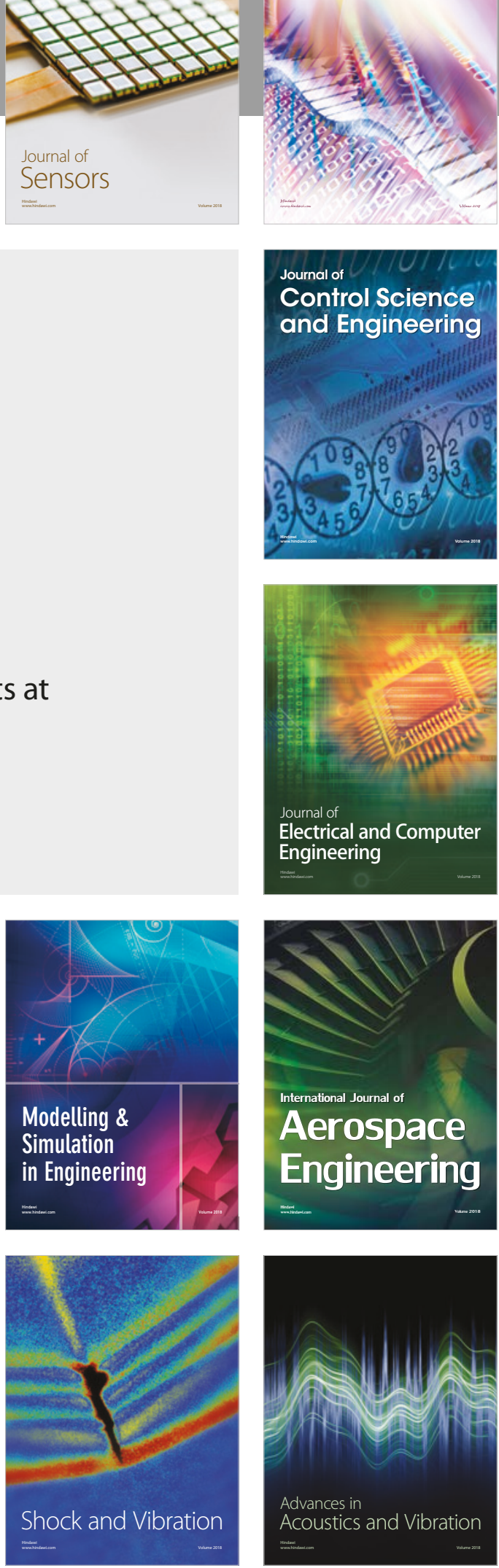